\title{
ON A PAPER OF PHELPS
}

\section{ROBERT SINE ${ }^{1}$}

In [4], Phelps showed that in certain function algebras the unit ball is the closed convex hull of its extreme points. The algebra, $C(X)$, of complex valued continuous functions on a compact Hausdorff space, will always have this property. The class of logmodular algebras which have a Gleason part which is total also was shown to have the property. In this paper we give an elementary proof of the first result (a proof which is, in theory at least, constructive). The simplest nontrivial example of a logmodular algebra with a total part is the disk algebra (i.e. the functions continuous on the closed disk and analytic in the interior). For this algebra we show that the extreme points (in fact the exposed points) of the unit ball form a dense subset of the boundary of the unit ball.

Let $U$ be the unit ball of $C(X)$. It is well known that $q$ in $U$ is an extreme point of $U$ iff $|q(x)|=1$ for all $x$ in $X$. Now if $f$ in $U$ never vanishes, then $f$ is in the closed convex hull of the extreme points; in fact $f$ is between two uniquely determined extreme points. We need only observe that for each $x$ in $X, f(x)$ is halfway between two uniquely determined extreme points of the disk and that these points vary continuously with $x$. Now it is not necessarily the case that each function in $U$ can be approximated by a nonvanishing function. For a counterexample we need only look at $h(z)=z$ in the algebra of continuous functions on the disk. (If $|f(z)-z| \leqq 1$, then any fixed point for the difference is a zero for $f$.) However if we can show that $h(z)=z$ can be approximated by convex combinations of extreme functions in $C$ of the disk, the problem will be completely solved. For if $g$ is in the ball of $C(X)$ and we can write $\left|z-\sum a_{i} q_{i}(z)\right|<\epsilon$ for $|z| \leqq 1$, then we can compose to get $\left|z \circ g(x)-\sum a_{i} q_{i} \circ g(x)\right|<\epsilon$ for all $x$ in $X$. Then to show $h(z)=z$ admits the desired approximation we pick $0<\epsilon<1$. We construct a retract map $f$ of the disk onto the disk with a wedge removed. The wedge should contain $z=0$ in its interior and have its vertex inside the disk $|z|<\epsilon$. We also take the wedge so narrow that there are $N$ rotations of the wedge which are disjoint in the annulus $\epsilon \leqq|z| \leqq 1$ where $N>1 / \epsilon$. Let $f_{i}, i=1, \cdots, N$ be the retract maps obtained from these rotations. Then $\left|1 / N \sum f_{i}(z)-z\right|$ $\leqq 2 \epsilon$ for $|z|<\epsilon$. If $\epsilon \leqq|z| \leqq 1$, then $f_{i}(z)=z$ for all but at most one value of $i$. We then obtain $2 / N \leqq 2 \epsilon$ as the estimate for $\epsilon \leqq|z| \leqq 1$.

Received by the editors January 20, 1966.

1 This work was supported by NSF Grant GP-4033. 
So $h(z)$ can be approximated by a convex combination of nonvanishing functions of modulus at most 1 ; each of these, in turn, is a convex combination of extreme functions.

If $X$ is not finite, $C(X)$ can be given an equivalent norm so that the unit ball of the new norm has no extremes. Pick $g$ to be any lower semicontinuous function on $X$ which satisfies $0<r<g(x)<s$ $<\infty$ for all $x$ in $X$. Let $K=\{f \in C(X):|f(x)| \leqq g(x)$ for all $x \in X\}$; the Minkowski functional of $K$ is an equivalent norm. Suppose $g$ is continuous. Rather than modify the above proof we can observe $f \rightarrow g f$ is a linear and topological automorphism of $C(X)$. Then $f$ is extreme in $K$ iff $|f|=g$, and $K$ is the closed convex hull of its extreme points. If $X$ is not finite, we can choose a discontinuous $g ; K$ then has no extremes.

Now we consider the disk algebra. If $f$ in the unit ball has the property that the set $\{|z|=1:|f(z)|=1\}$ has positive measure then $f$ is extreme. For if $f=1 / 2\left(g_{1}+g_{2}\right)$, then $g_{1}-g_{2}=0$ on a set of the circumference of positive measure. Actually the condition holds iff $f$ is an exposed point of the ball; see [4]. We will not need this last fact. We will need the following result of Bieberbach from conformal mapping [see 1, p. 136 or 2 , p. 181]. Let $R$ be a simply connected region with a rectifiable boundary whose boundary curve is of length $L$. Let $|L-2 \pi|<\epsilon \cdot 2 \pi$ where $0<\epsilon<1$ and suppose that the boundary curve lies entirely in the annulus formed by the two concentric circles whose center is at $z=0$ and whose radii are $1-\epsilon$ and $1+\epsilon$. Let $f$ be the analytic function that gives a conformal mapping of $|z|<1$ onto $R$ and that satisfies $f(0)=0$ and $f^{\prime}(0)<0$. Then the following relation holds in $|z| \leqq 1 ;|f(z)-z|<(2 \cdot 2) \pi(2 \epsilon)^{1 / 2}$.

Theorem. The set of points of the unit sphere $S$ of the above form are dense in $S$.

Proof. Let $\Delta(1)$ be the closed unit disk. Given $f$ in $S$, we can approximate $f$ by a polynomial $P$ so that $\|P\|_{\infty}=1$. Now $P(\Delta)$ is a Jordan domain (at least upon taking the union of $P(\Delta)$ with any bounded components of the complement). Let $\Delta(r)$ be the disk of radius $r<1$ and set $R(r)=P(\Delta) \cup \Delta(r)$. Take $r<1$ so large that the length of the boundary of $R(r)$ is within $2 \pi \epsilon$ of $2 \pi$. Then take $r$ even larger if necessary to obtain also $r \geqq 1-\epsilon$. (We can take $r<1$ still larger if necessary so that $R(r)$ is simply connected; in this case the plugging of the holes mentioned above is not necessary to obtain a Jordan domain.) Then by the Bieberbach Theorem there is a conformal map $\phi$ (the inverse of the map $f$ of the theorem) of $R(r)$ onto the closed unit disk which is an approximation of the identity map. 
Then $\phi \circ P$ is in $S$ and approximates $f$. The map $\phi$ extends to a homeomorphism of the boundaries by the well known Osgood-TaylorCaratheodory extension of the Riemann mapping theorem $[3, \mathrm{p}$. $130]$. Now the set of the circumference, $\{s:|\phi \circ P(s)|=1\}$, will in fact contain an arc; for $\phi$ is bicontinuous and the polynomial is locally bicontinuous. Thus $\{|z|=1:|\phi \circ P(z)|=1\}$ has positive measure.

From the density of extreme points in $S$, it follows at once that $U$ is the closed convex hull of its extreme points.

\section{BiBLIOGRAPHY}

1. L. Bieberbach, Conformal mapping, Chelsea, New York, 1964.

2. - S.-B. Sitzungsber. Preuss. Akad. Wiss. (1924).

3. M. Heins, Selected topics in the classical theory of functions of a complex variable, Holt, Rinehart, and Winston, New York, 1952.

4. R. Phelps, Extreme points in function algebras, Duke Math. J. 32 (1965), 267277.

University of Minnesota 